\title{
INVERSE PROBLEMS IN FOURIER ANALYSIS AND SUMMABILITY THEORY*
}

\author{
MARK A. PINSKY ${ }^{\dagger}$
}

Key words. Fourier analysis, Summability theory

AMS subject classifications. 41A25, 42A50

1. Introduction. The oldest theorems of Fourier analysis give sufficient conditions under which

$$
\lim _{M \rightarrow \infty} S_{M} f(x)=\frac{1}{2}[f(x+0)+f(x-0)] .
$$

Here $f$ is an integrable function on the circle and $S_{M} f$ is the associated Fourier partial sum. This can be viewed as an inverse problem, namely to determine the local average of a function, given the knowledge of its Fourier partial sums. These conditions can be substantially relaxed if the Fourier partial sum is replaced by a suitable summability method.

One can also ask to obtain the jump from the Fourier partial sums, for example by studying the derivative $\left(S_{M} f\right)^{\prime}(x)$. This inverse problem is more delicate, since the localization principle of Riemann is not valid for the derivatives of the partial sums. Nevertheless in 1913 Fejér $[\mathrm{F}]$ found that, assuming suitable regularity

$$
\lim _{M \rightarrow \infty} \frac{\left(S_{M} f\right)^{\prime}(x)}{M}=C_{1}[f(x+0)-f(x-0)]
$$

where $C_{1}$ is a universal constant, whose value depends on the convention used to define $S_{M} f$. Results of this type also hold for various summability procedures and were studied by Lukács [L], Zygmund [Z] and others. In all of these works the jump in (2) may be replaced by a suitable local average jump whose precise definition depends on the regularity of the summability method.

Instead of using the derivative to retrieve the jump, one may also use the conjugate partial sum $\tilde{S}_{M} f$. In this case one obtains a logarithmic behavior, leading to a result of the form

$$
\lim _{M \rightarrow \infty} \frac{\left(\tilde{S}_{M} f\right)(x)}{\log M}=C_{2}[f(x+0)-f(x-0)]
$$

for another universal constant. Results of this type were obtained by Fejér $[\mathrm{F}]$ and Lukács [L] for the Fourier partial sum and by Móricz[M2] for Abel summability of Fourier series on the circle.

The purpose of this paper is to give a unified treatment of these results for functions on the line. The corresponding results on the circle can be obtained by periodization techniques. When we come to the analysis of the conjugate function, it is most efficient to use the definition of the conjugate Poisson integral formulated by Koosis[Ko], which is simultaneously defined on all of the Lebesgue spaces $L^{p}(R), 1 \leq p \leq \infty$.

\footnotetext{
*Received March 16, 2004; accepted for publication September 1, 2004.

${ }^{\dagger}$ Mathematics Department, Northwestern University, 2033 Sheridan Road, Evanston, IL 602082730, USA (pinsky@math.northwestern.edu).
} 
2. Analysis of the Fourier partial sum. The simplest instance of (2) occurs when we consider a function on the real line. Let $f \in L^{1}(R)$ and define the Fourier transform and Fourier partial sum by

$$
\hat{f}(u)=\int_{R} e^{-2 \pi i u y} f(y) d y, \quad S_{M} f(x)=\int_{-M}^{M} e^{2 \pi i u x} \hat{f}(u) d u .
$$

Theorem 2.1. Suppose that $f \in L^{1}(R) \cap B V(R)$. Then for all $x \in R$,

$$
M^{-1} \frac{d}{d x}\left(S_{M} f\right)(x) \rightarrow 2(f(x+0)-f(x-0)), \quad M \uparrow \infty .
$$

First we state and prove an elementary fact:

Lemma 2.2. For any $f \in L^{1}(R) \cap B V(R), \lim _{x \rightarrow \pm \infty} f(x)=0$.

Proof. For any $a<b$, we have

$$
\begin{gathered}
f(b)-f(a)=\int_{(a, b]} d f \\
|f(b)-f(a)| \leq \int_{(a, b]}|d f|
\end{gathered}
$$

which tends to zero when $a, b \rightarrow \infty$ or $a, b \rightarrow-\infty$, by the dominated convergence theorem. This proves that the $\operatorname{limits}_{\lim } \lim _{x \rightarrow \pm \infty} f(x)$ exist. But $f \in L^{1}(R)$ implies that both limits are zero.

Proof of the theorem. Computing directly, we have

$$
\begin{aligned}
S_{M} f(x) & =\int_{-M}^{M}\left(\int_{R} e^{2 \pi i u(x-y)} f(y) d y\right) d u \\
& =2 \int_{0}^{M}\left(\int_{R} \cos 2 \pi u(x-y) f(y) d y\right) d u \\
\frac{d}{d x} S_{M} f(x) & =-2 \int_{0}^{M} 2 \pi u\left(\int_{R} \sin 2 \pi u(x-y) f(y) d y\right) d u \\
& =-2 \int_{0}^{M}\left(\int_{R} \frac{d}{d y}(\cos 2 \pi u(x-y)) f(y) d y\right) d u \\
& =+2 \int_{0}^{M}\left(\int_{R} \cos 2 \pi u(x-y) d f(y)\right) d u \\
& =2 \int_{R} \frac{\sin 2 \pi M(x-y)}{2 \pi(x-y)} d f(y)
\end{aligned}
$$

where we have used the lemma to discard the terms at $\pm \infty$ in the partial integration. Now the measure $d f$ has finite total mass, while the integrand is bounded and has the value $M$ when $y=x$. Dividing both sides by $M$, we obtain

$$
\lim _{M} M^{-1} \frac{d}{d x}\left(S_{M} f\right)(x):=2 d f(\{x\})=2[f(x+0)-f(x-0)]
$$

which was to be proved. 
2.1. A refined result. If the jump $d f(\{x\})$ is zero, we can obtained a more refined asymptotic formula for $S_{M} f^{\prime}(0)$ in terms of jumps at other points. This is closely related to the Pinsky phenomenon [Ka] which was first studied in the context of multi-dimensional Fourier analysis $[\mathrm{P}]$.

ThEOREM 2.3. Suppose that $y \rightarrow f_{x}(y):=f(x+y)-f(x-y)$ is piecewise absolutely continuous with $f_{x}^{\prime} \in L^{1}(R)$ and $f_{x}\left(0^{+}\right)=0$. Assume in addition that $y \rightarrow f_{x}^{\prime}(y)$ satisfies a Dini condition at $y=0$. Then

$$
\left(S_{M} f\right)^{\prime}(x)=\sum_{j=1}^{K} \frac{\sin 2 \pi M t_{j}}{\pi t_{j}} \Delta_{j}+\frac{1}{2}\left[f^{\prime}(x+0)+f^{\prime}(x-0)\right]+o(1), \quad M \rightarrow \infty
$$

where $\left(t_{j}\right)_{1 \leq j \leq K}$ are the non-zero jump points of $y \rightarrow f_{x}(y)$ and $\Delta_{j}=f_{x}\left(t_{j}+0\right)-f_{x}\left(t_{j}-0\right), 1 \leq j \leq K$.

This result gives an explicit expression of the failure of Riemann localization for the derivative of the partial sums.

Proof. It is no loss of generality to do the case $x=0$, since we can always replace $f(t)$ by $f(t \pm x)$. From the previous computation, we have

$$
\begin{aligned}
\left(S_{M} f\right)^{\prime}(0) & =-\int_{R} f(y) \frac{d}{d y}\left(\frac{\sin 2 \pi M y}{\pi y}\right) d y \\
& =-\int_{0}^{\infty}[f(y)-f(-y)] \frac{d}{d y}\left(\frac{\sin 2 \pi M y}{\pi y}\right) d y .
\end{aligned}
$$

We can integrate-by-parts on each finite interval $[a, b]$ on which $f$ is absolutely continuous:

$$
\begin{aligned}
\int_{a}^{b}[f(y)-f(-y)] \frac{d}{d y}\left(\frac{\sin 2 \pi M y}{\pi y}\right) & =[f(y)-f(-y)]\left(\frac{\sin 2 \pi M y}{\pi y}\right) \mid \begin{array}{l}
y=b \\
y=a
\end{array} \\
& -\int_{a}^{b}\left[f^{\prime}(y)+f^{\prime}(-y)\right] \frac{\sin 2 \pi M y}{\pi y} d y .
\end{aligned}
$$

If $a \neq 0, b \neq 0$, each of the endpoint contributions gives the stated contribution, while the new integral tends to zero, by the Riemann-Lebesgue lemma. In case $a=0$ or $b=0$, the new integral is the standard Fourier partial sum for $f_{x}^{\prime}$, which satisfies a Dini condition at $y=0$. Hence this new integral converges to the stated value when $M \rightarrow \infty$.

2.2. The conjugate Fourier partial sum. The conjugate Fourier partial sum is analyzed in a similar fashion. By definition

$$
\begin{aligned}
\tilde{S}_{M} f(x): & =-i \int_{0}^{M} e^{2 \pi i u x} \hat{f}(u) d u+i \int_{-M}^{0} e^{2 \pi i u x} \hat{f}(u) d u \\
& =\int_{R} \frac{1-\cos 2 \pi M(x-y)}{\pi(x-y)} f(y) d y
\end{aligned}
$$

where we have used the definition of $\hat{f}$ and evaluated $\int_{0}^{M}$ and $\int_{-M}^{0}$.

THEOREM 2.4. Suppose that $f \in L^{\infty}(R) \cap L^{1}(R)$ and there exists $\delta_{0}(f, x):=$ $f(x+0)-f(x-0)$. Then

$$
\frac{\tilde{S}_{M} f(x)}{\log M} \rightarrow-\frac{1}{\pi}[f(x+0)-f(x-0)], \quad M \uparrow \infty .
$$


Proof. The conjugate Fourier partial sum is written

$$
\begin{aligned}
\tilde{S}_{M} f(x) & =\int_{R} \frac{1-\cos 2 \pi M t}{\pi t} f(x-t) d t \\
& =\int_{0}^{\infty} \frac{1-\cos 2 \pi M t}{\pi t}[f(x-t)-f(x+t)] d t
\end{aligned}
$$

Note that for any $R_{0}>0$,

$$
\int_{0}^{R_{0}} \frac{1-\cos 2 \pi M t}{\pi t} d t=\int_{0}^{M R_{0}} \frac{1-\cos 2 \pi t}{\pi t} d t=\frac{1}{\pi} \log M+O(1)
$$

Letting $F(t)=f(x-t)-f(x+t)+\delta_{0}(f, x)$, we have for any $R_{0}>0$,

$$
\tilde{S}_{M} f(x)+\frac{\delta_{0}(f, x)}{\pi} \log M=\int_{0}^{R_{0}} F(t) \frac{1-\cos 2 \pi M t}{\pi t} d t+O(1) .
$$

Given $\epsilon>0$, choose $\delta>0$ so that $|F(t)|<\epsilon$ for $|t|<\delta$. Then

$$
\begin{aligned}
& \left|\int_{0}^{\delta} F(t) \frac{1-\cos 2 \pi M t}{\pi t} d t\right| \leq \epsilon \int_{0}^{\delta} \frac{1-\cos 2 \pi M t}{\pi t} d t<\epsilon \log M \\
& \left|\int_{\delta}^{R_{0}} F(t) \frac{1-\cos 2 \pi M t}{\pi t} d t\right|<\|F\|_{\infty} \int_{\delta}^{R_{0}} \frac{2}{\pi t} d t \leq\|F\|_{\infty} \log \left(R_{0} / \delta\right)=O(1) .
\end{aligned}
$$

Dividing both sides of (8) by $\log M$, we have

$$
\underset{M}{\limsup }\left|\frac{\tilde{S}_{M} f(x)}{\log M}+\frac{\delta_{0}(f, x)}{\pi}\right|<\epsilon
$$

which was to be proved.

It is interesting to note that this result for the conjugate partial sum requires no more smoothness hypotheses than the existence of the jump $\delta_{0}(f, x)$. By contrast, the result for the derivative in Theorem 2.1 requires that $f$ be of bounded variation. This discrepancy in assumptions is consistent with the classical results of Lukács [L] for Fourier series on the circle.

2.3. Fejér's Gibbs phenomenon. Fejér also discovered the following Gibbslike phenomenon for the jump. There exists a universal sequence $x_{M} \downarrow 0$, so that for any $f$ above,

$$
\lim _{M \rightarrow \infty}\left[S_{M} f\left(x+x_{M}\right)-S_{M} f\left(x-x_{M}\right)\right]=f(x+0)-f(x-0) .
$$

Here $x_{M}$ may be chosen as the smallest positive root $x$ of the equation

$$
\int_{x}^{\infty} \frac{\sin 2 \pi M t}{\pi t} d t=0
$$

We will not pursue this theme in this paper. 
3. The generalized jump of a function. For a locally integrable function on the real line we can consider three different notions of jump. We set

$$
\begin{aligned}
& \delta_{0}(f, x)=\lim _{t \downarrow 0}(f(x+t)-f(x-t)) \\
& \delta_{2}(f, x)=\lim _{h \downarrow 0} \frac{1}{h} \int_{0}^{h}(f(x+t)-f(x-t)) d t
\end{aligned}
$$

provided that these limits exist. An intermediate notion is the existence of $\delta_{1}(f, x)$ with the property that

$$
\lim _{h \downarrow 0} \frac{1}{h} \int_{0}^{h}\left|f(x+t)-f(x-t)-\delta_{1}(f, x)\right| d t=0 .
$$

If $\delta_{0}(f, x)$ exists, then so does $\delta_{1}(f, x)$ and they are equal. If $\delta_{1}(f, x)$ exists, then so does $\delta_{2}(f, x)$ and they are equal. At the end of this section we give the proof that for a.e. $x, \delta_{1}(f, x)$ exists and is zero, generalizing a well-known result for functions of bounded variation.

In the next paragraph we provide examples of locally integrable functions for which

- a) None of the three limits exist.

- b) Only $\delta_{2}(f, 0)$ exists.

- c) Only $\delta_{1}(f, 0)$ and $\delta_{2}(f, 0)$ exist.

To see this, begin with the locally integrable function defined for $t>0$ by

$$
f(t)=t^{\alpha} \sin \left(\frac{1}{t^{\beta}}\right), \quad \alpha>-1, \beta>0
$$

where we set $f(t)=0$ for $t \leq 0$. If $\alpha>0$, then $\delta_{0}(f, 0)=0$. We claim that $\delta_{2}(f, 0)$ exists if and only if $\alpha+\beta>0$, whereas $\delta_{1}(f, 0)$ exists if and only if $\alpha>0$. To see this make the change of variable $s=1 / t^{\beta}$ and write

$$
\begin{aligned}
\int_{0}^{h} t^{\alpha} \sin \left(\frac{1}{t^{\beta}}\right) d t & =\frac{1}{\beta} \int_{h^{-\beta}}^{\infty} \frac{\sin s}{s^{\alpha / \beta+1+1 / \beta}} d s \\
& =-\frac{1}{\beta} \int_{h^{-\beta}}^{\infty} s^{-[\alpha / \beta+1+1 / \beta]} d(\cos s) \\
& =\frac{1}{\beta}\left(h^{-\beta}\right)^{-1-\alpha / \beta-1 / \beta}\left(\cos \left(h^{-\beta}\right)+o(1)\right) \\
& =\frac{1}{\beta} h^{\alpha+\beta+1}\left(\cos \left(h^{-\beta}\right)+o(1)\right) \\
\frac{1}{h} \int_{0}^{h} f(t) d t & =\frac{1}{\beta} h^{\alpha+\beta}\left(\cos \left(h^{-\beta}\right)+o(1)\right), \quad h \downarrow 0 .
\end{aligned}
$$

If $\alpha+\beta>0$, then $\delta_{2}(f, 0)=0$. If $\alpha+\beta \leq 0$, then $\delta_{2}(f, 0)$ does not exist.

To compute $\delta_{1}(f, 0)$ for this example, note from the above that if it exists it must be zero. However we can compute directly as follows: 


$$
\begin{aligned}
\int_{0}^{h} t^{\alpha}\left|\sin \left(\frac{1}{t^{\beta}}\right)\right| d t & =\frac{1}{\beta} \int_{h^{-\beta}}^{\infty} \frac{|\sin s|}{s^{\alpha / \beta+1+1 / \beta}} d s \\
& \sim \operatorname{const} \sum_{\left[h^{-\beta}\right]}^{\infty} \frac{1}{n^{1+1 / \beta+\alpha / \beta}} \\
& =\operatorname{const} h^{1+\alpha}(1+o(1)) \\
\frac{1}{h} \int_{0}^{h}|f(t)| d t & \sim \operatorname{const} h^{\alpha}(1+o(1)), \quad h \downarrow 0 .
\end{aligned}
$$

Thus we see that if $\alpha>0$ then $\delta_{1}(f, 0)$ exists and is zero. If $\alpha \leq 0$ and $\delta_{1}(f, 0)$ did exist, then it must be zero, since $\delta_{2}(f, 0)=0$. But the above analysis shows that the choice $\delta_{1}(f, 0)=0$ gives a contradiction.

To summarize, the above class of examples show the possibilities a) and b). Finally we give an example for which only $\delta_{1}(f, 0)$ and $\delta_{2}(f, 0)$ exist. Let

$$
f(t)=1, \quad\left|t-2^{-n}\right| \leq \frac{3^{-n}}{2} \quad n=1,2, \ldots
$$

and $f(t)=0$ otherwise. These intervals are non-overlapping and the integral on each interval is $3^{-n}$. Hence

$$
0 \leq t \leq 2^{-N} \Longrightarrow \int_{0}^{t} f(s) d s \leq \sum_{n=N}^{\infty} 3^{-n}=\frac{3}{2} 3^{-N} .
$$

Therefore if $2^{-(N+1)} \leq t \leq 2^{-N}$

$$
\frac{1}{t} \int_{0}^{t} f(s) d s \leq 2^{N+1} \frac{3}{2} 3^{-N}=3\left(\frac{2}{3}\right)^{N}
$$

which tends to zero when $t \downarrow 0$. But clearly $\lim \sup _{t \rightarrow 0} f(t)=+1$, hence $\delta_{0}(f, 0)$ does not exist but $\delta_{1}(f, 0)=0=\delta_{2}(f, 0)$.

3.1. Proof that $\delta_{1}(f, x)=0$ a.e. This follows closely the details of the proof of the Lebesgue differentiation theorem using the Hardy-Littlewood maximal function. If $f$ is a continuous function, then clearly $\delta_{1}(f, x)=0$ for every $x \in R$. For any $f \in L^{1}(R)$ and $\epsilon>0$, there exists a continuous function $g$ such that $\|f-g\|_{1}<\epsilon$. Let $f=g+r$ and set

$$
N f(x, h):=\frac{1}{h} \int_{0}^{h}|f(x+t)-f(x-t)| d t \leq N g(x, h)+N r(x, h) .
$$

Since $\lim _{h \downarrow 0} N g(x, h)=0$, we have

$$
\limsup _{h \downarrow 0} N f(x, h) \leq \limsup _{h \downarrow 0} N r(x, h) \leq \sup _{h>0} \frac{1}{h} \int_{x-h}^{x+h}|r(t)| d t \leq 2 M r(x)
$$

where $M r$ is the Hardy-Littlewood maximal function of $r$. Hence for any $\delta>0$

$$
\begin{aligned}
\left|\left\{x: \limsup _{h \downarrow 0} N f(x, h)>\delta\right\}\right| & \leq|\{x: 2 M r(x)>\delta\}| \\
& \leq \frac{C}{\delta}\|r\|_{1} \\
& \leq \frac{C}{\delta} \epsilon
\end{aligned}
$$


where the Hardy-Littlewood maximal inequality was applied in the second line. But $\epsilon>0$ was arbitrary. Hence the Lebesgue measure on the left side must be zero, for any $\delta>0$. This means that $\lim _{h \downarrow 0} N f(x, h)=0$ a.e. for any $f \in L^{1}$. If $f$ is only locally integrable, then apply the above argument on a sequence of compact sets whose union is the real line, which completes the proof.

4. Extension to summability kernels. In this section we extend Theorem 2.1, where we replace the Fourier partial sum by a convolution operator with a suitable class of kernels. Specifically, we consider a family of integral transforms on the real line, written

$$
K_{y} f(x)=\int_{R} f(x-y t) k(t) d t, \quad y>0 .
$$

In every case, $k$ is an absolutely continuous real-valued function with

$$
k \in L^{1}(R), \quad k^{\prime} \in L^{1}(R), \quad k(-t)=k(t), \quad \forall t \in R .
$$

Note that we do not require that the integral of $k$ be normalized to 1 . This normalization would be convenient in studying $\lim _{y \downarrow 0} K_{y} f(x)$ but is not relevant in studying the derivative $\left(K_{y} f\right)^{\prime}(x)$ when $y \downarrow 0$. In addition we consider the following properties:

$$
\left|k^{\prime}(t)\right| \leq L(t), \forall t>0 \text {, where } L \in L^{1}\left(R^{+}\right) \text {is monotone decreasing }
$$

$k^{\prime}$ is absolutely continuous with $k^{\prime}(t) \leq 0, k^{\prime \prime}(t) \geq 0$ for $t \geq t_{0}>0$.

For examples the Poisson kernel with $k(t)=1 / \pi\left(1+t^{2}\right)$ and the Gauss kernel with $k(t)=e^{-\pi t^{2}}$ satisfy all three properties. The Fejér kernel with $k(t)=(1-\cos t) / \pi t^{2}$ satisfies (15) and (16) but not (17). In general, any kernel that satisfies (15) and (17) also satisfies (16). Recalling the definitions of the jumps $\delta_{i}(f, x)$ from the previous section, we have the following three results for the derivative approximations.

TheOREm 4.1. Suppose that $k$ satisfies (15). Let $f \in L^{\infty}(R)$ and suppose that for some $x \in R, \delta_{0}(f, x)$ exists. Then $\left(K_{y} f\right)^{\prime}(x)$ exists for $y>0, x \in R$ and

$$
\lim _{y \downarrow 0} y\left(K_{y} f\right)^{\prime}(x)=k(0) \delta_{0}(f, x) .
$$

This result can be considered a counterpart of Theorem 2.1 on the derived Fourier integral.

Theorem 4.2. Suppose that $k$ satisfies (15) and (16). Let $f \in L^{\infty}(R)$ and suppose that for some $x \in R, \delta_{1}(f, x)$ exists. Then

$$
\lim _{y \downarrow 0} y\left(K_{y} f\right)^{\prime}(x)=k(0) \delta_{1}(f, x) .
$$

This result generalizes Zygmund's theorem to the present setting, when Lebesguetype conditions hold. In order to handle the case of more general points, we require a more stringent condition on the kernel.

Theorem 4.3. Suppose that $k$ satisfies (15), (16) and (17). Let $f \in L^{\infty}(R)$ and suppose that for some $x \in R, \delta_{2}(f, x)$ exists. Then

$$
\lim _{y \downarrow 0} y\left(K_{y} f\right)^{\prime}(x)=k(0) \delta_{2}(f, x) .
$$


4.1. Proofs. We begin by writing

$$
K_{y} f(x)=\int_{R} f(t) k\left(\frac{x-t}{y}\right) \frac{d t}{y} .
$$

Hypothesis (15) allows one to differentiate the integral to obtain

$$
\begin{aligned}
\left(K_{y} f\right)^{\prime}(x):=\frac{d}{d x} K_{y} f(x) & =\int_{R} f(t) k^{\prime}\left(\frac{x-t}{y}\right) \frac{d t}{y^{2}} \\
& =\frac{1}{y} \int_{R} f(x-y t) k^{\prime}(t) d t .
\end{aligned}
$$

From (15), $k^{\prime}$ is an odd function, so that we can write

$$
\begin{gathered}
\left(K_{y} f\right)^{\prime}(x)=\frac{1}{y} \int_{0}^{\infty}[f(x-y t)-f(x+y t)] k^{\prime}(t) d t \\
y\left(K_{y} f\right)^{\prime}(x)-k(0) \delta_{0}(f, x)=\int_{0}^{\infty}\left[f(x-y t)-f(x+y t)+\delta_{0}(f, x)\right] k^{\prime}(t) d t .
\end{gathered}
$$

To prove Theorem 4.1, we note that $k^{\prime} \in L^{1}(R)$ so that given $\epsilon>0$ we may choose $M>0$ so that $\int_{M}^{\infty}\left|k^{\prime}(t)\right| d t<\epsilon$. From (10) we see that the integral on the interval $[0, M]$ tends to zero by the dominated convergence theorem, so that Theorem 4.1 follows.

To proceed further we state and prove a useful lemma.

Lemma 4.4. Suppose that $j(t)$ is defined for $t \geq t_{0}$ with $\int_{t_{0}}^{\infty}|j(t)| d t<\infty$ so that $j(t) \leq 0$ and $j^{\prime}(t) \geq 0$ for $t \geq t_{0}$. Then $\int_{t_{0}}^{\infty} t j^{\prime}(t) d t<\infty$.

Proof. Under these hypotheses, we can write

$$
t j(t)-t_{0} j\left(t_{0}\right)=\int_{t_{0}}^{t} j(s) d s+\int_{t_{0}}^{t} s j^{\prime}(s) d s
$$

When $t \rightarrow \infty$ the first integral has a finite limit while the second integral tends to some $C \in[0, \infty]$. Hence there exists $D=\lim _{t \rightarrow \infty} t j(t),-\infty<D \leq \infty$. But $j(t) \leq 0$ implies that $D \leq 0$. But $D \neq 0$ is impossible, since $\int_{t_{0}}^{\infty}|j(t)| d t<\infty$. We have proved that $\int_{t_{0}}^{\infty} s j^{\prime}(s) d s=-t_{0} j\left(t_{0}\right)-\int_{t_{0}}^{\infty} j(s) d s<\infty$, as required.

To prove Theorem 4.2 , we write

$$
\begin{aligned}
E: & =y\left(K_{y} f\right)^{\prime}(x)-k(0) \delta_{1}(f, x) \\
\Phi(t) & =\int_{0}^{t}\left|f(x-u)-f(x+u)+\delta_{1}(f, x)\right| d u
\end{aligned}
$$

so that $\Phi(t) / t \rightarrow 0$ when $t \rightarrow 0$. Finally, let $\epsilon(t)=\Phi(t) / t$, a bounded function with 
$\lim _{t \downarrow 0} \epsilon(t)=0$, we have

$$
\begin{aligned}
|E| & \leq \int_{0}^{\infty}|f(x-t y)-f(x+t y)+\delta(x)|\left|k^{\prime}(t)\right| d t \\
& \leq \int_{0}^{\infty} L(t) \Phi^{\prime}(t y) d t \\
& =\int_{0}^{\infty} \frac{L(t)}{y} \frac{d}{d t}[\Phi(t y)] d t \\
& =-\int_{0}^{\infty} \frac{\Phi(t y)}{y} L^{\prime}(t) d t \\
& =-\int_{0}^{\infty} \epsilon(t y)\left(t L^{\prime}(t)\right) d t .
\end{aligned}
$$

But Lemma 4.4 applied to $t_{0}=0, j(t)=-L(t)$ shows that $-t L^{\prime}(t)$ is the density of a finite measure on $[0, \infty)$; but the integrand tends to zero boundedly when $y \rightarrow 0$ so that the result follows by the dominated convergence theorem.

To prove Theorem 4.3, define $\Psi(t)=\int_{0}^{t}\left(f(x-u)-f(x+u)+\delta_{2}(f, x)\right) d u$ and $\epsilon(t)=\Psi(t) / t$, a bounded function with $\lim _{t \rightarrow 0} \epsilon(t)=0$. Then

$$
\begin{aligned}
E:=y\left(K_{y} f\right)^{\prime}(x)-k(0) \delta_{2}(f, x) & =\int_{0}^{\infty}\left[f(x-y t)-f(x+t y)+\delta_{2}(f, x)\right] k^{\prime}(t) d t \\
& =\int_{0}^{\infty} k^{\prime}(t) \Psi^{\prime}(t y) d t \\
& =-\int_{0}^{\infty} \frac{1}{y} \Psi(t y) k^{\prime \prime}(t) d t \\
& =\left(\int_{0}^{t_{0}}+\int_{t_{0}}^{\infty}\right) t k^{\prime \prime}(t) \epsilon(t y) d t \\
& :=J_{1}+J_{2} .
\end{aligned}
$$

Then

$$
\left|J_{1}\right| \leq \sup _{0 \leq t \leq y t_{0}}|\epsilon(u)| \int_{0}^{t_{0}} t\left|k^{\prime \prime}(t)\right| d t
$$

which tends to zero when $y \rightarrow 0$. On the interval $\left(t_{0}, \infty\right), t \rightarrow t k^{\prime \prime}(t)$ is a non-negative integrable function, while $\epsilon(t y) \rightarrow 0$ boundedly when $y \downarrow 0$. Therefore $J_{2} \rightarrow 0$ by the dominated convergence theorem. This completes the proof of the first set of results.

5. Summability of the conjugate partial sum. In parallel with the direct summability methods involving the derivative, one can model a class of kernels based on the conjugate partial sum. We consider kernels $k(t)$ satisfying

$$
k(-t)=-k(t), \quad 0 \leq t k(t) \leq C, \quad \lim _{t \rightarrow \infty} t k(t)=k_{\infty} .
$$

This includes the conjugate Poisson kernel where $k(t)=t /\left[\pi\left(1+t^{2}\right)\right]$ and the conjugate Fejér kernel, where $k(t)=(\pi t)^{-1}\left(1-\frac{\sin t}{t}\right)$. (see section 6.2). Note that neither of these kernels is integrable. In the appendix we give the detailed computation of the conjugate Fejér kernel. In general, we define a sequence of linear functionals

$$
L_{M} f=\int_{R} M k(M t) f(t) d t
$$


and recall the definitions of $\delta_{0}, \delta_{1}, \delta_{2}$ in $(10),(11),(12)$. Then

TheOREM 5.1. Suppose that $f \in L^{1}(R, d x /(1+|x|))$ and that $\delta_{0}(f, 0)$ exists.

$$
\lim _{M \rightarrow \infty} \frac{L_{M} f}{\log M}=k_{\infty} \delta_{0}(f, 0)
$$

TheOREm 5.2. Suppose, in addition to (22), we have $|k(t)| \leq K(t)$ for $t \geq 0$, where $K$ is monotone increasing for $0 \leq t \leq y_{0}$, monotone decreasing for $t \geq y_{0}$ and satisfies (22). Suppose that $f \in L^{1}(R, d x /(1+|x|))$ and that $\delta_{1}(f, 0)$ exists. Then

$$
\lim _{M \rightarrow \infty} \frac{L_{M} f}{\log M}=k_{\infty} \delta_{1}(f, 0) .
$$

TheOREM 5.3. Suppose, in addition to (22), that $k$ is monotone increasing for $0 \leq t \leq y_{0}$ and monotone decreasing for $t \geq y_{0}$. Suppose that $f \in L^{1}(R, d x /(1+|x|))$ and that $\delta_{2}(f, 0)$ exists. Then

$$
\lim _{M \rightarrow \infty} \frac{L_{M} f}{\log M}=k_{\infty} \delta_{2}(f, 0) .
$$

These three theorems give successively weaker conditions on $f$ while imposing increasingly stronger conditions on the kernel $k$. It is also understood that these results are to be applied to a convolution operator $f \rightarrow \int_{R} f(x-t y) k(t)$ at $x=0$.

\subsection{Proofs.}

Proof of Theorem 5.1. Write

$$
L_{M} f=\int_{0}^{\infty}[f(t)-f(-t)] M k(M t) d t
$$

Given $\epsilon>0$, choose $\eta>0$ so that $\left|f(t)-f(-t)-\delta_{0}(f, 0)\right|<\epsilon$ for $0<t<\eta$. Then

$$
\begin{aligned}
L_{M} f & =\left(\int_{0}^{\eta}+\int_{\eta}^{\infty}\right)=I+I I \\
|I I| & \leq \sup _{0 \leq z}|z k(z)| \int_{\eta}^{\infty}\left|\frac{f(t)-f(-t)}{t}\right| d t=O(1) \\
\left|I-\delta_{0}(f, 0) \int_{0}^{\eta} M k(M t) d t\right| & =\left|\int_{0}^{\eta}\left[f(y)-f(-y)-\delta_{0}(f, 0)\right] M k(M y) d y\right| \\
& <\epsilon \int_{0}^{\eta} M k(M y) d y \\
& =\epsilon \int_{0}^{M \eta} k(z) d z \\
& <\epsilon(\log M+O(1)) .
\end{aligned}
$$

But $\int_{0}^{\eta} M k(M t) d t=k_{\infty} \log M+O(1), M \rightarrow \infty$. Thus

$$
\left|K_{M} f-\delta_{0}(f, 0) k_{\infty} \log M\right|<\epsilon \log M+O(1) .
$$


Dividing by $\log M$ and letting $M \rightarrow \infty$ gives the result.

Coming to the proofs of the Theorems 5.2 and 5.3, we will need to integrate by parts and deal with the contributions from the differentiated terms. These will be handled by a simple lemma.

Lemma 5.4. For any $K$ satisfying the hypothesis of Theorem 5.2 and $\eta>0$,

$$
\int_{0}^{\eta} z M^{2}\left|K^{\prime}(M z)\right| d z \leq C_{1}+C_{2} \log M
$$

Proof. For $M>y_{0} / \delta$, we can write the integral in two portions, corresponding to the interval $\left(0, y_{0} / M\right)$ and the interval $\left(y_{0} / M, \eta\right)$. In the first case we have

$$
\begin{aligned}
\int_{0}^{y_{0} / M} z M^{2}\left|K^{\prime}(M z)\right| d z & =y_{0} K\left(y_{0}\right)-\int_{0}^{y_{0} / M} M K(M z) d z \\
& =y_{0} K\left(y_{0}\right)-\int_{0}^{y_{0}} K(u) d u=O(1) .
\end{aligned}
$$

In the second case, we have

$$
\begin{aligned}
\int_{y_{0} / M}^{\eta} z M^{2}\left|K^{\prime}(M z)\right| d z & =y_{0} K\left(y_{0}\right)-M \eta K(M \eta)+\int_{y_{0} / M}^{\eta} M K(M u) d u \\
& =O(1)+\int_{y_{0}}^{M \eta} K(u) d u \\
& =O(1)+O(\log M)
\end{aligned}
$$

which completes the proof.

Proof of Theorem 5.2. Let $F(t)=\int_{0}^{t}\left|f(z)-f(-z)-\delta_{1}(f, 0)\right| d z$. For any $\eta>0$, write

$$
\begin{array}{r}
K_{M} f-\delta_{1}(f, 0) \int_{0}^{\eta} M k(M t) d t=\int_{0}^{\eta}\left[f(t)-f(-t)-\delta_{1}(f, 0)\right] M k(M t) d t \\
+\int_{\eta}^{\infty}[f(t)-f(-t)] M k(M t) d t
\end{array}
$$

The second integral is handled in exactly the same way as in Theorem 5.1. To handle the first integral, we can write for $M>y_{0} / \eta$,

$$
\begin{aligned}
& \left|\int_{0}^{\eta}\left[f(t)-f(-t)-\delta_{1}(f, 0)\right] M k(M t) d z\right| \leq \int_{0}^{\eta} M K(M t) F^{\prime}(t) d z \\
= & M K(M \eta) F(\eta)-\int_{0}^{\eta} M^{2} K(M z) F(z) d z .
\end{aligned}
$$

Given $\epsilon>0$, choose $\eta>0$ so that $|F(z) / z|<\epsilon$ for $0<z<\eta$. Then the first term is less than $\epsilon C$. The new integral is estimated by Lemma 5.4 by $\epsilon\left(C_{1}+C_{2} \log M\right)$. Summarizing, we have the estimate

$$
K_{M} f-\delta_{0}(f, 0) \int_{0}^{\eta} M k(M t) d t=O(1)+\epsilon\left(C_{1}+C_{2} \log M\right) .
$$


Dividing by $\log M$ and taking $M \rightarrow \infty$ produces the result.

Proof of Theorem 5.3. Let $G(t)=\int_{0}^{t}\left[f(z)-f(-z)-\delta_{2}(f, 0)\right] d z$, so that $G(t) / t \rightarrow$ 0 . Following the previous steps, we have

$$
L_{M} f-\delta_{2}(f, 0) \int_{0}^{\eta} M k(M t) d t=O(1)+\int_{0}^{\eta} M k(M t) G^{\prime}(t) d t .
$$

In this case the kernel $k$ satisfies the hypothesis satisfied by $K$ in Theorem 5.2. Hence we can integrate by parts and follow the steps in the previous proof. $\square$

5.2. The conjugate Poisson kernel. Theorem 5.3 applies to the conjugate Poisson integral, defined classically [SW] for $f \in L^{1}(R)$ as

$$
-Q_{y} f(x)=\frac{1}{\pi} \int_{R} \frac{t-x}{(t-x)^{2}+y^{2}} f(t) d t .
$$

In order to obtain a more generally applicable theory, we follow Koosis [Ko] and add a constant to consider

$$
-\tilde{Q}_{y} f(x)=\frac{1}{\pi} \int_{R}\left(\frac{t-x}{(t-x)^{2}+y^{2}}-\frac{t}{1+t^{2}}\right) f(t) d t .
$$

If $f \in L^{p}(R)$ for some $1 \leq p<\infty$ we can separate the two terms to see that $Q_{y} f(x)-\tilde{Q}_{y} f(x)$ is a constant. In general $\tilde{Q}_{y} f(x)$ is defined on a larger space, namely

$$
B_{2}=\left\{f:\|f\|:=\int_{R} \frac{|f(x)|}{1+x^{2}} d x<\infty\right\} .
$$

Koosis $[\mathrm{K}]$ has shown that for any $f \in B_{2}$ there exists a.e. the conjugate function $\tilde{H} f(x)=\lim _{y \downarrow} \tilde{Q}_{y} f(x)$ and we have the Kolmogorov inequality

$$
\frac{1}{\pi} \int_{\{x:|\tilde{H} f(x)|>\alpha\}} \frac{d x}{1+x^{2}} \leq \frac{4}{\alpha}\|f\| \quad \alpha>0 .
$$

Corollary 5.5. Suppose that $f \in B_{2}$ and that for some $x \in R, \delta_{2}(f, x)$ exists. Then

$$
\lim _{y \downarrow 0} \frac{\tilde{Q}_{y} f(x)}{\log (1 / y)}=-\frac{1}{\pi} \delta_{2}(f, x) .
$$

Proof. We change variables to $u=x-t$ which leads to

$$
\begin{aligned}
\tilde{Q}_{y} f(x) & =\frac{1}{\pi} \int_{R} \frac{u^{2} x+u\left(1+x^{2}-y^{2}\right)-x y^{2}}{\left(u^{2}+y^{2}\right)\left(1+(x+u)^{2}\right)} f(x+u) d u \\
& =I+I I+I I I .
\end{aligned}
$$

To estimate $I$, we discard $y^{2}$ in the denominator to obtain

$$
|I| \leq \frac{|x|}{\pi} \int_{R} \frac{|f(x+u)|}{1+(x+u)^{2}} d u=O(1)
$$


which is independent of $y$. Similary to estimate $I I I$ we discard $u^{2}$ in the denominator to obtain

$$
|I I I| \leq \frac{|x|}{\pi} \int_{R} \frac{f(x+u)}{1+(x+u)^{2}} d u=O(1) .
$$

It remains to analyze

$$
I I:=\frac{1+x^{2}-y^{2}}{\pi} \int_{R} \frac{u}{\left(u^{2}+y^{2}\right)\left(1+(x+u)^{2}\right.} f(x+u) d u .
$$

The function $u \rightarrow f(x+u) /\left(1+(x+u)^{2}\right)$ satsifies the hypotheses of Theorem 5.3, as does the conjugate Poisson kernel $\pi k(t)=t /\left(1+t^{2}\right)$. Hence we can apply Theorem 5.3 to conclude that $I I / \log (1 / y) \rightarrow(1 / \pi) \delta_{2}(f, x)$

\section{Appendix.}

6.1. Proof of (21). In the absence of a published reference, we provide a direct proof of the elementary fact that for $f \in L^{\infty}(R)$ and $k, k^{\prime} \in L^{1}(R)$ we can differentiate under the sign of integration.

Without loss of generality, we can make a change-of-variable and assume that $y=1, x=0$. We write the difference quotient:

$$
\begin{aligned}
\frac{1}{x}\left(K_{1} f(x)-K_{1} f(0)\right) & =\frac{1}{x} \int_{R} f(t)(k(x-t)-k(-t)) d t \\
& =\frac{1}{x} \int_{R} f(t)\left(\int_{-t}^{x-t} k^{\prime}(u) d u\right) d t \\
& =\int_{R} k^{\prime}(u)\left(\frac{1}{x} \int_{-u}^{-u+x} f(t) d t\right) d u
\end{aligned}
$$

where we have interchanged the orders of integration in the last step. The new integrand is bounded pointwise by $\|f\|_{\infty}$ and converges a.e. to $f(-u)$. Since $k^{\prime} \in$ $L^{1}(R)$, we can apply the Lebesgue dominated convergence theorem to conclude that

$$
\left.\frac{d}{d x} K_{1} f(x)\right|_{x=0}=\int_{R} k^{\prime}(-u) f(u) d u
$$

as required.

6.2. The conjugate Fejér kernel. Since it is not easy to find a reference, we include here the detailed computation of the conjugate Fejér kernel. This is defined in terms of its Fourier transform for $x \neq 0$ :

$$
\begin{aligned}
\tilde{K}_{M}(x) & =i \int_{-M}^{0}\left(1+\frac{\xi}{M}\right) e^{2 \pi i \xi x} d \xi-i \int_{0}^{M}\left(1-\frac{\xi}{M}\right) e^{2 \pi i \xi x} d \xi \\
& =i \int_{-M}^{0}\left(1+\frac{\xi}{M}\right) d\left(\frac{e^{2 \pi i \xi x}}{2 \pi i x}\right)-i \int_{0}^{M}\left(1-\frac{\xi}{M}\right) d\left(\frac{e^{2 \pi i \xi x}}{2 \pi i x}\right) \\
& =i\left(\frac{1}{2 \pi i x}-\frac{1}{M} \int_{-M}^{0} \frac{e^{2 \pi i \xi x}}{2 \pi i x} d \xi\right)-i\left(-\frac{1}{2 \pi i x}+\frac{1}{M} \int_{0}^{M} \frac{e^{2 \pi i \xi x}}{2 \pi i x} d \xi\right) \\
& =\frac{1}{2 \pi x}-\frac{i}{2 \pi M x}\left(\frac{1-e^{-2 \pi i M x}}{2 \pi i x}\right)+\frac{1}{2 \pi x}-\frac{i}{2 \pi M x}\left(\frac{e^{2 \pi i M x}-1}{2 \pi i x}\right) \\
& =\frac{1}{\pi x}\left(1-\frac{\sin 2 \pi M x}{2 \pi M x}\right)
\end{aligned}
$$


which is non-negative for $x \geq 0$ and satisfies the conditions that $x \tilde{K}_{M}(x) \leq \frac{2}{\pi}$ and $\lim _{x \rightarrow \infty} x \tilde{K}_{M}(x)=\frac{1}{\pi}$.

\section{REFERENCES}

[F] L. FejÉR, Uber die Bestimmung des Sprunges der Funktion aus ihrer Fourierreihe, Journal für die Reine und Angewandte Mathematik, 142 (1913), pp. 165-188.

[Ka] J. P. Kahane, Le phénomène de Pinsky et la géométrie des surfaces, CRAS Paris, 321 (1995), pp. 1027-1029.

[Ko] P. Koosis, The Logarithmic Integral I, Second Edition, Cambridge University, Press 1998.

[L] F. LuKÁcs, Uber die Bestimmung des Sprunges der Funktion aus ihrer Fourierreihe, Journal für die Reine und Angewandte Mathematik, 150 (1920), pp. 107-112.

[M1] F. MóRICZ, Extension of a theorem of Fejér to double Fourier-Stieltjes series, Journal of Fourier Analysis and Applications, 7 (2001), pp. 601-614.

[M2] F. Móricz, Ferenc Lukács type theorems in terms of the Abel-Poisson mean of conjugate series, Proceeedings of the American Mathematical Society, 131 (2003), pp. 1243-1250.

[P] M. PInsky, Pointwise Fourier inversion and related eigenfunction expansions, Communications in Pure and Applied Mathematics, 47 (1994), pp. 653-681.

[SW] E.M. Stein And G. Weiss, Introduction to Fourier Analysis on Euclidean Spaces, Princeton University Press, 1971.

[Z] A. Zygmund, Trigonometric Series, volume 1, Cambridge University Press, Cambridge, UK, 1959. 